\title{
Safe airflow education in a brave new world
}

EMS and the Swiss Dental Academy (SDA) have announced that their popular Guided Biofilm Therapy (GBT) events are back on track, with new protocols to ensure everyone's safety.

The courses are ideal for dental hygienists and therapists looking to offer their patients the very best in prophylaxis and to renew their vigour for dentistry in these challenging times, while achieving a financial benefit.

Currently, the SDA is offering the GBT Masterclass, a half-day of theoretical knowledge to develop strategies for periodontal and implant maintenance by looking at the components of the GBT model including AirFlow, PerioFlow and the advancements in 'No Pain' Piezon technology.

The GBT protocol is based on individual patient diagnosis and risk assessment in order to achieve optimal results. The treatment is given in the least invasive way, with the highest level of comfort, safety and efficiency.

\section{A powerful disinfectant}

OptiZil is a powerful disinfectant that helps dental practices clean surfaces up to five times faster than products with a five-minute contact time. The nontoxic sanitiser is proven effective against 99.9999\% of bacteria and viruses in '1-minute' and approved for BS EN14476 (virucidal test).

OptiZil multi-surface disinfectant is part of the CleanCert Innovations $360^{\circ}$ approach to effective clinical infection control. The range also includes CleanCert waterline cleaner and biofilm remover and OraWize+ antimicrobial mouthwash.

The active ingredient in every product is hypochlorous acid ( $\mathrm{HOCl})$, a natural biocide that is exceptionally potent yet non-toxic, non-carcinogenic, or a mucosa irritant.

Thanks to an innovative manufacturing process, the hypochlorous in OptiZil, CleanCert and OraWize+ is stabilised

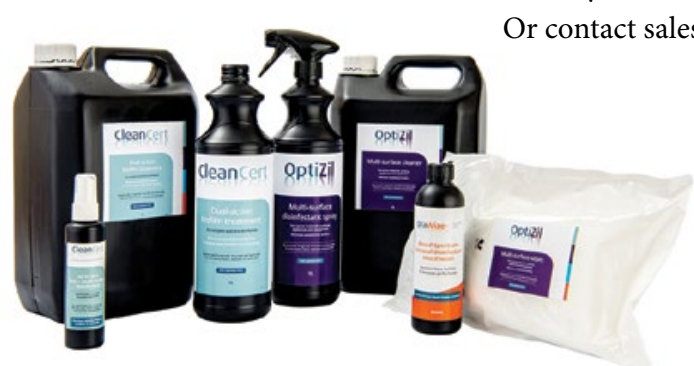

This means that even previously anxious patients are happy to return time and again, boosting practice income on a regular basis.

Course numbers are limited to ten, in a space suitable to hold at least 20 delegates, so that social distancing can be easily achieved. The organiser will also be printing QR codes for track and trace, keeping windows and doors open, plus providing hand sanitiser on arrival and masks to whoever wants them.

SDA trainer Faye Donald is back in the SDA saddle along with Sally Simpson, Amanda Gallie and Christina Chatfield, and new recruits Anna Middleton, Lottie Manahan and Benjamin Tighe. They are excited to share their knowledge and love of the GBT protocol with others.

For further information on upcoming events and locations and to book, visit https://bit.ly/30lKZPh.

In the event of any local lockdowns, a full refund will be given.

\section{Balancing business with} clinical excellence

Streamline your workflow and enhance patient care with Carestream Dental's innovative Sensei Cloud - the latest true cloud-based practice management platform.

Acting as a trusted digital mentor, Sensei Cloud is optimised to analyse your practice data, report on key performance indicators, and provide actionable steps that you can take to boost revenue.

It combines an intuitive design with powerful imaging, practice management and clinical workflow capabilities in one cloud-based platform, which can be accessed anywhere, any time for ultimate flexibility.

With real-time dashboards, revenue cycle management, built-in scalability and a variety of other invaluable tools, Sensei Cloud enables you to balance business with clinical excellence.

For more information please contact Carestream Dental on 08001699692 or visit www.carestreamdental.co.uk.

For all the latest news and updates, follow us on Twitter@CarestreamDentl and Facebook.

1. Fast-acting. The one-minute contact time helps turnaround dental stations and environments much quicker in the fallow time

2. No pre-mixing required. Staff save time because our products are pre-mixed and ready to use straight from the container

3. No need to spend time on staff training. No need to train staff in hazardous chemical use as OptiZil, CleanCert and OraWize+ are biocompatible and non-toxic

4. Benefit from bulk-buy discounts. The 12-month shelf life means practices can buy in bulk to save money without worrying about products losing efficacy.

Ask your distributor about special offers. Or contact sales@cleancert.co.uk. 